\title{
Targeting gastrointestinal stromal tumors: the role of regorafenib
}

This article was published in the following Dove Press journal:

OncoTargets and Therapy

20 May 2016

Number of times this article has been viewed

\author{
Brett Schroeder' \\ Zula $\mathrm{Li}^{2,3}$ \\ Lee D Cranmer ${ }^{2,3}$ \\ Robin L Jones ${ }^{4}$ \\ Seth M Pollack ${ }^{2,3}$ \\ 'College of Human Medicine, Michigan \\ State University, Grand Rapids, MI, \\ ${ }^{2}$ Division of Medical Oncology, \\ University of Washington, ${ }^{3} \mathrm{Clinical}$ \\ Research Division, Fred Hutchinson \\ Cancer Research Center, Seattle,WA, \\ USA; ${ }^{4}$ Royal Marsden Hospital, \\ Institute of Cancer Research, \\ London, UK
}

\begin{abstract}
Gastrointestinal stromal tumor (GIST) is a devastating disease in the metastatic setting, but its natural history has been dramatically altered by the development of small molecule tyrosine kinase inhibitors, most notably imatinib. Although patients with advanced GIST live much longer today than they did in the past, imatinib-refractory disease remains a tremendous problem. For disease that is refractory to imatinib and sunitinib, regorafenib is an excellent option. In this review, we discuss the biology and clinical work establishing regorafenib as the standard of care for advanced GIST refractory to both imatinib and sunitinib.
\end{abstract}

Keywords: regorafenib, GIST, refractory, imatinib

\section{Introduction to gastrointestinal stromal tumors}

Gastrointestinal stromal tumor (GIST) is one of the most common soft tissue sarcoma subtypes; each year $\sim 3,300-6,000$ new cases are diagnosed. ${ }^{1,2}$ They occur largely in middle-aged and elderly persons, with $\sim 60 \%$ located in the stomach, $30 \%$ in the small intestine, and $10 \%$ in other regions of the gastrointestinal (GI) tract. ${ }^{3}$ Size, mitotic rate, and location of the primary lesion are the most important prognostic factors. ${ }^{4}$

The initial presentation of patients with GIST often reflects the underlying biology of the tumor. GISTs are often highly vascularized and may be relatively large at presentation. GI bleeding occurs in more than half of the patients; a palpable mass is present in one-third of the patients. Pain, in some cases due to obstruction, occurs in nearly one-quarter of the patients. ${ }^{5}$ Nonspecific presenting symptoms are common, such as early satiety or bloating.

Because of the initial symptoms related to abdominal pathology, computed tomography (CT) is a frequent initial imaging modality in patients with GIST. Triple-phase CT (intravenous and oral contrast with venous and arterial phases) allows observation of the extent and vascularity of these tumors, and assesses for hepatic involvement. ${ }^{5}$ GISTs typically metastasize to the liver and GI tract, although dissemination to other sites, notably the lungs, is also possible. CT imaging of these regions can provide staging information in these areas.

While clinical and radiographic findings may be suggestive, the definitive diagnosis of GIST relies on tissue diagnosis. Histologically, GIST can have a variable appearance, but has been classified in the past as frequently displaying features of smooth muscle tumors. A key observation in GIST diagnosis and therapy was the central role of mitogen receptor and GIST marker, c-Kit; gain-of-function of this receptor is crucial for tumor growth in most cases of GISTs. ${ }^{6}$ Overexpression of c-Kit protein is detected immunohistochemically in the majority of GISTs, which is a key diagnostic finding. 
GIST can also occur from mutations in the plateletderived growth factor receptor- $\alpha$ (PDGFRA) gene. So-called wild-type GISTs lack mutations in both KIT, and PDGFRA. ${ }^{7}$ The GIST morphology ranges from predominantly spindleshaped (70\%), epithelioid (20\%), to a mixed phenotype $(10 \%){ }^{8,9}$ A minor subset (5\%) of GISTs are c-Kit negative, and these often occur in the stomach with an epithelioid cell morphology and a usually less aggressive course. ${ }^{10}$ DOG1 (ANO1) is expressed in GISTs regardless of KIT expression and supports the diagnosis.

In patients with primary and localized GIST, the standard therapy is surgical resection with the intention to cure. In the pre imatinibera, approximately one-half of the patients diagnosed with localized GIST would relapse and patients with recurrent, unresectable, and/or metastatic GIST would die in their first year following diagnosis. Today, complete resection for primary GIST is associated with a 5-year recurrence-free survival rate of $70 \% .{ }^{11}$ Image-guided core needle biopsy is also useful in diagnostic evaluation and should be done first if GIST is suspected in the case of a large tumor and where neoadjuvant imatinib is being considered. ${ }^{4}$

\section{Tyrosine kinase inhibitors have revolutionized the treatment of GIST}

In 1998, investigators made a critical discovery showing that gain-of-function mutations in the proto-oncogene for the tyrosine kinase receptor c-Kit drive GISTs. ${ }^{12}$ These mutations are present in over $85 \%$ of GISTs and are able to induce tumor growth in vitro and in vivo. ${ }^{13,14}$ Exon 11 encodes the protein's juxtamembrane domain and is the most common location for mutations. Mutations of exon 11 permit ligand-independent receptor activation and independent tumor growth. ${ }^{15,16}$ The prognostic significance associated with different KIT mutations can be profound; exon 11 KIT mutations yield $\sim 89 \%$ 5-year survival versus $40 \%$ for other mutation types. ${ }^{13,16,17}$ Most KIT mutations are found in exons $9,11,13$, or 17 .

A treatment breakthrough occurred in 2001, when the first case report was published describing a patient with GIST who had successfully been treated with the tyrosine kinase inhibitor (TKI), imatinib. This patient had rapidly progressive, metastatic GIST. The patient progressed despite multiple prior systemic therapies (dacarbazine, doxorubicin, ifosfamide, interferon- $\alpha$, and thalidomide). Imatinib led to a complete metabolic response within 1 month of treatment initiation. ${ }^{18}$ The same year, researchers assembled 36 patients with GIST with advanced disease to evaluate the safety and efficacy of imatinib. ${ }^{19}$ Minor responses were elicited in
$17 \%$ (six of 36 ) of patients, while partial remissions were achieved in $53 \%$ (19 of 36 ) of patients.

In 2002, 147 patients with advanced GIST were enrolled in a multicenter Phase II trial in which they were randomized to receive either 400 or $600 \mathrm{mg}$ of imatinib daily. The patients had undergone a number of previous treatment modalities: $15 \%$ received radiotherapy, 51\% received chemotherapy, and $98 \%$ had a previous operation. No patient achieved a complete response, but 54\% (79 patients) had partial response and 28\% (41 patients) had stable disease (treatment benefit lasting $>6$ months). Neither dose was superior, although the study was underpowered to detect a difference at 52-month follow-up. ${ }^{20}$ Subsequent studies have continued to document the efficacy of imatinib in both the metastatic and adjuvant settings. ${ }^{19-23}$

Imatinib has become the gold standard for GIST therapy, as it provides a stable response, typically for 18-36 months. Prior to the introduction of imatinib, a median survival time of 10-20 months was generally seen. Today, this has improved to 51-57 months. ${ }^{24}$ Fortunately, imatinib has a manageable toxicity profile. Common side effects include diarrhea, nausea, headache, edema, myalgia, rash, and some abdominal discomfort.

Despite the vast improvement in GIST therapy with the advent of imatinib, the drug is not curative for patients with metastatic disease, and resistance ultimately emerges. Investigation of planned salvage therapies has been undertaken. For example, the multitargeted TKI sunitinib has been approved for treatment of patients with GIST after progression on imatinib therapy. Sunitinib, improves progression-free survival (PFS) in imatinib-refractory patients with GIST, particularly so in patients with exon 9 KIT mutations. ${ }^{25}$ Sunitinib can also be given on the continuous daily dosing schedule without apparent loss of efficacy. ${ }^{25}$

\section{Regorafenib is a small molecule TKI that also inhibits angiogenesis}

GIST biology has proven to be more complex than originally believed, as evidenced by the molecular heterogeneity found in all GISTs and the numerous subgroups identified. ${ }^{26}$ These tumors are distinct from nerve sheath tumors or GI smooth muscle tumors, and may originate in the interstitial cells of Cajal. ${ }^{27}$ Activating mutations of c-KIT are not sufficient to cause GIST; additional genomic alterations are present, but their exact biological contribution remain largely unknown. ${ }^{28-30}$

The success of imatinib and sunitinib raised interest in a wide variety of multitargeted small molecule TKIs that affect c-Kit to varying degrees, such as dasatinib and sorafenib. ${ }^{31,32}$ 
During this period of TKI investigation, regorafenib's potent inhibitory activity was recognized. ${ }^{33-35}$ While orally available and structurally similar to sorafenib, regorafenib is a TKI against multiple targets important for tumor angiogenesis, oncogenesis, and overall maintenance of the tumor microenvironment (Figure 1). ${ }^{33}$ Vascular endothelial growth factor receptor 2 (VEGFR-2) and other tyrosine kinases with immunoglobulin and epidermal growth factor homology domain 2 are critical to the biology of both normal and tumor vasculatures, but have been targeted successfully in cancer. While angiogenesis is a well-recognized component of tumor development and has been demonstrated to be an important therapeutic target in many malignancies, ${ }^{36,37}$ its role in GIST was largely underappreciated despite the frequent vascularity of these tumors. Similar to tumor growth, angiogenesis is governed by interconnected tyrosine kinase-driven signaling pathways, which are affected by other small molecule TKI GIST therapies, including sunitinib and sorafenib.

Similar to other small molecule TKIs, regorafenib has direct inhibitory effects on tumor growth through inhibition of KIT, as well as Ras and B-Rapidly Accelerated Fibrosarcoma (BRAF) downstream of Platelet Derived Growth Factor Receptor Beta (PDGFR $\beta) .{ }^{38}$ For patients with some imatinib resistance mediated by secondary KIT mutations, direct inhibition of c-Kit by regorafenib may play a particularly important role. ${ }^{39}$

Interestingly, in colorectal cancer (CRC) cell lines, regorafenib has been demonstrated as an important agonist of the tumor suppressor Src Homology region 2 domain containing Phosphatase-1 (SHP-1). ${ }^{40}$ Regorafenib may also alter mitogen activated protein (MAP) kinases, Extracellular signal Regulated Kinase (ERK) pathway, and c-Jun N-terminal Kinase (JNK/Jun) in complex ways that are incompletely characterized. ${ }^{41-43}$ In other systems, increased cytosolic $\mathrm{Ca}^{++}$ may play a role. ${ }^{44}$ Regorafenib directly impairs mitochondrial function by uncoupling oxidative phosphorylation, which may play a role in rare reports of hepatic toxicity. ${ }^{45}$

Unlike imatinib, and to a greater degree than other small molecule TKIs, regorafenib inhibits endothelial cells based on biochemical and cellular kinase proliferation assays by targeting VEGFR-2 and TIE-2. ${ }^{33}$ This additional activity allows regorafenib to maintain activity even in xenograft models of GIST that are highly resistant to c-Kit targeting. ${ }^{39}$ Similar explanations can be invoked for the use of regorafenib in GIST.

VEGFR targeting is mechanistically important, as regorafenib improves overall survival in patients with metastatic, treatment-refractory CRCs. ${ }^{47,48}$ Given its similarity to pazopanib, which improves PFS in soft tissue sarcoma patients, ${ }^{49,50}$ regorafenib is now being broadly tested in soft tissue sarcomas. ${ }^{51}$

\section{Regorafenib has a toxicity profile acceptable for most patients with refractory metastatic GIST}

In the first reported Phase I trial of regorafenib, the drug was used in patients with advanced solid tumors refractory to

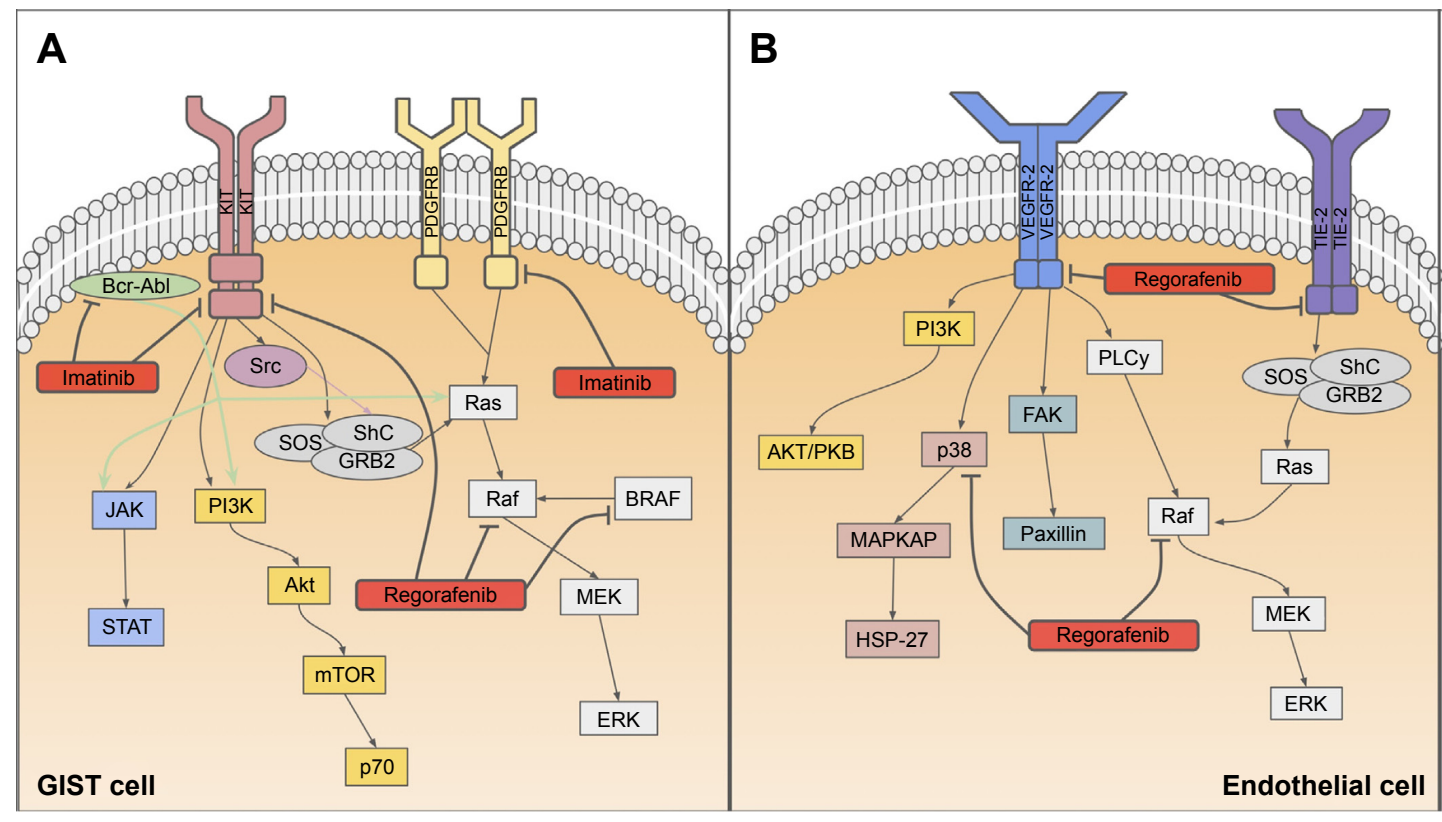

Figure I In the gastro-intestinal stromal tumor cell (A), regorafenib inhibits phosphorylation of the KIT receptor and activation of Raf and BRAF. Imatinib targets the KIT and PDGF receptors, as well as the Bcr-Abl kinase. In the endothelial cell (B), regorafenib restricts signal transduction at the VEGF and TIE receptors, in addition to the P38 and Raf kinases specifically.

Abbreviation: GIST, gastrointestinal stromal tumor. 
standard chemotherapy. A total of 53 patients were recruited for this study to assess the drug's safety, pharmacokinetics, pharmacodynamics, and efficacy profile. ${ }^{34}$ Dosing on the Phase I study ranged from 10 to $220 \mathrm{mg} /$ day on a 21 of 28-day oral cycle. While five of 12 patients treated at the $220 \mathrm{mg} /$ day dosing experienced toxicities, none were lifethreatening. The $160 \mathrm{mg} /$ day dosing schedule was deemed superior based on its toxicity profile (two of 12 patients at this dose had dose-limiting toxicity) and was selected for further investigation.

Surprisingly, given a highly refractory and diverse patient population, $66 \%$ of these patients experienced disease control (ie, partial response or stable disease). An expanded Phase I cohort analyzed the safety in metastatic CRC. ${ }^{52}$ Thirty-eight patients were enrolled with heavily pretreated CRC (median number of previous therapies was 4). Of the patients eligible for evaluation, $70 \%$ (19 of 27) were found to have stable disease; pharmacodynamic assessment demonstrated decreased tumor perfusion in most patients. ${ }^{52}$

The safety of regorafenib has been tested extensively, and its toxicity profile is well established and consistent (Table 1). ${ }^{47,48,53}$ Like other VEGFR-targeted TKIs, regorafenib can induce hypertension, requiring additional antihypertensive therapy. ${ }^{54}$ Another common toxicity of regorafenib in some patients is hand-foot skin reactions (HFSRs). HFSR is not as frequent in patients on sunitinib, but grade 3 or higher HFSR has been observed in all the randomized controlled trials of regorafenib. Maculopapular rashes and generalized desquamation can also occur, but are rarely severe. ${ }^{48}$ Diarrhea occurs in $<10 \%$ of patients treated with regorafenib. It is generally not severe and can be responsive to Lomotil (diphenoxylate and atropine). Although rare, regorafenib has been associated with fatal (grade 5) toxicities, including cardiac arrest and hepatic failure. ${ }^{53}$
There is also an association between regorafenib therapy and an increased incidence of myocardial ischemia, potentially linked to the inhibition of VEGF pertaining to its role in cardiovascular function. Patients with preexisting cardiovascular comorbidities, including venous thrombus, embolic events such as stroke or transient ischemic attacks, pulmonary embolus, cardiac arrhythmias requiring antiarrhythmic therapy, uncontrolled hypertension despite optical medical management, or severe heart failure within 6 months before the start of treatment were excluded from the trial. Although a Phase I trial assessing the cardiovascular safety of regorafenib in solid tumors showed the drug to have modest effects on QT/QTc and left ventricular ejection fraction. ${ }^{55}$ In $72 \%$ of patients treated with regorafenib, the drug dosage had to be modified due to adverse events. However, the incidence of adverse events leading to permanent drug discontinuation was $6.1 \%$ in the regorafenib arm, similar to the $7.6 \%$ in the placebo-treated patients. ${ }^{53,56}$

Despite some potentially serious toxicities, regorafenib is well tolerated in the vast majority of patients. ${ }^{55}$ Patients participating in a randomized Phase III trial of regorafenib were given a quality-of-life survey. There was no significant difference in the quality-of-life between patients treated with regorafenib and those receiving best supportive care, though quality-of-life worsened after disease progression. ${ }^{57}$

\section{Regorafenib improves PFS for patients with refractory metastatic GIST}

The preliminary efficacy from the Phase I study was further evaluated in a multicenter, Phase II study of regorafenib in 33 patients. These patients had previously been treated with both imatinib and sunitinib, yet they progressed, or they were unable to tolerate the treatments. Twenty-six patients

Table I Grades 3 and 4 toxicities reported by the GRID and CORRECT trials, grade 5 was reported by GRID only

\begin{tabular}{lllllll}
\hline Toxicity & \multicolumn{2}{l}{ GRID } & & & \multicolumn{2}{c}{ CORRECT* } \\
\cline { 2 - 3 } \cline { 5 - 6 } & Grade 3 (\%) & Grade 4 (\%) & Grade 5 (\%) & & Grade 3 (\%) & Grade 4 (\%) \\
\hline Any & 58 & 2 & - & - & 51 & 17 \\
HFSR & 20 & 0 & - & 7 & 0 \\
Hypertension & 23 & 1 & - & 7 & 0 \\
Diarrhea & 5 & 0 & - & 9 & $<1$ \\
Fatigue & 2 & 0 & - & 3 & $<1$ \\
Thrombocytopenia & - & - & - & 2 & - \\
Anemia & - & - & $<1$ & - & - \\
Cardiac arrest & - & - & $<1$ & - & - \\
Hepatic failure & - & - & &
\end{tabular}

Note: *The CORRECT trial used regorafenib in the treatment of colorectal cancer, not gastrointestinal tumors.

Abbreviations: GRID, GIST-Regorafenib In progressive Disease; HFSR, hand-foot skin reaction; CORRECT, regorafenib monotherapy for previously treated metastatic colorectal cancer. 
(75\%) derived clinical benefit from regorafenib, as evidenced by either a partial or complete tumor response, or stable disease for a minimum of 16 weeks. ${ }^{35}$ Most patients (22 of 26) had demonstrated stable disease, but four exhibited partial responses by response evaluation criteria in solid tumors (RECIST). The lack of substantial tumor remission in a majority of treated patients, as has been seen with other TKIs in the treatment of GIST, might suggest that alternative response assessment criteria should be used.

PFS for the entire cohort on the Phase II study was 10 months (95\% confidence interval, 8.3-14.9 months). Patients with exon 11 KIT mutations experienced longer PFS versus those with exon 9 mutations. However, this was an exploratory analysis based on a small sample and may be secondary to the relative aggressiveness of the two mutational domains, rather than any increased associated response to therapy. Clinical responses were seen despite dose reductions in a majority of patients for grade 3 toxicities affecting their quality-of-life.

These results led to the GIST-Regorafenib In progressive Disease (GRID) trial, an international, multicenter, randomized, placebo-controlled, Phase III trial with 199 patients who had either metastatic or unresectable disease. Furthermore, these patients previously had unsuccessful treatment with imatinib (understood as progression or intolerance to treatment), and were unsuccessfully treated with sunitinib (identified by disease progression). ${ }^{53}$ Patients were randomized using 2-to-1 to treatment with either regorafenib or placebo. All patients also received best supportive care (defined as any method to preserve the comfort and dignity of the patient, excluding disease-specific antineoplastic therapy). Oral regorafenib treatment was given at the same dose and schedule as the Phase II trial, with $160 \mathrm{mg}$ /day taken for 21 days of each 28-day cycle.

The primary endpoint of the study was PFS, which was 4.8 months for those who received regorafenib, versus 0.9 months for those who received placebo (hazard ratio $=0.27 ; P<0.00001)$. No patient in either group demonstrated a complete response. The disease control rate (defined as rate of complete or partial response plus stable disease lasting at least 12 weeks) was $52.6 \%$ (70 of 133) for regorafenib and $9.1 \%$ (six of 66 ) for placebo ( $95 \%$ confidence interval, 54.72-32.49; $P<0.00001)$.

There was no difference in the overall survival between the two arms. The study was not intended to detect a survival benefit; crossover from the placebo group to the active treatment was allowed, with $85 \%$ of placebo-treated patients ultimately receiving regorafenib at the time of progression. Toxicity in the GRID trial (Table 1) was similar to regorafenib toxicities seen in other trials, including HFSR ( $56.1 \%$ vs $15.2 \%$, regorafenib vs placebo), hypertension $(48.5 \%$ vs $16.7 \%)$, diarrhea ( $40.9 \%$ vs $7.6 \%$ ), and oral mucositis (37.9\% vs $9.1 \%)$.

\section{Future directions}

Regorafenib is now approved for the treatment of GIST in the US and Europe and is considered by most experts to be the standard-of-care third-line agent for patients with metastatic or unresectable GIST that is refractory to imatinib and sunitinib. The impressive clinical activity in this highly refractory patient population raises the question as to whether regorafenib's use as first- or second-line treatment would be beneficial. In light of the established benefits, including overall survival benefits, or imatinib therapy, the design of clinical trials to test this hypothesis may present some challenges.

Given the multiple kinase targets of regorafenib (Figure 1), patients treated with regorafenib as a first-line agent may be less prone to resistance; this proposition merits further investigation as it may provide further support for testing regorafenib earlier in the GIST treatment continuum. A point of interest is why some patients with KIT mutations, such as exon 17, tend to have resistance to nilotninb, ${ }^{46,58}$ yet they show response to regorafenib. ${ }^{39}$ An ongoing trial is evaluating the activity of regorafenib in patients with a secondary mutation in exon 17 (NCT 02606097). Trials testing the role of regorafenib in the curative setting either as an alternative to imatinib or in combination with imatinib are compelling, particularly in the neoadjuvant setting where significant questions remain regarding which patients benefit most from imatinib treatment and for how long therapy should continue.

Regorafenib-refractory disease remains a critical problem, but a number of different strategies are under investigation. New TKIs, such as ponatinib (NCT 01874665), are under investigation. ${ }^{59,60}$ Combining small molecule TKIs along with other novel agents may allow treatment to overcome resistance. ${ }^{61}$ The observation that PI3 kinases (PI3K) and mechanistic target of rapamycin (MTOR) are upregulated in imatinib-resistant GIST lines ${ }^{62,63}$ led to the dose-finding study of the novel PI3K inhibitor, BKM120, in combination with imatinib (NCT 01468688). For some patients, BRAF resistance may play a role in imatinib resistance and targeting this pathway may play a role in treatment. ${ }^{64}$ Small molecule TKIs may have increased activity when combined with each other such as imatinib and sorafenib. ${ }^{65}$ The ongoing SURE trial is evaluating an alternating schedule of regorafenib and sunitinib (NCT 02164240). 
Monoclonal antibodies recognizing KIT are able to control imatinib-refractory GIST in xenograft models. ${ }^{66}$ These antibodies may directly slow GIST and enable macrophage-induced tumor clearance. These infiltrating immune and stromal cells play a critical role in the immune response to GIST, and the combination of TKI with novel immunotherapies is another source of great excitement in the field. ${ }^{20,23,40,67,68}$

Understanding genetic signatures and complex signaling networks are crucial for yielding successful outcomes using targeted therapies. Since regorafenib targets multiple kinases, further investigation of the interplay between oncogenesis and angiogenesis may explain treatment successes, as well as failures. One challenge that remains is cancer resistance to drug intervention, which inevitably leads to progression and a fatal outcome. In an era of next-generation sequencing, longitudinal molecular monitoring may allow us to understand better the evolution of drug resistance against current and future regimens. Study of regorafenib-treated patients may be especially pertinent, as most regorafenibtreated patients have been previously exposed to other agents (ie, imatinib and sunitinib). Combinations with other molecularly targeted agents, such as MTOR inhibitors, may unlock crucial synergies allowing patients to maintain efficacy for much longer.

Therapy of GIST continues to be an exciting field of investigation, and regorafenib has been an important addition to our anti-GIST armamentarium. Its use has generated important questions, the answers to which may improve the use of regorafenib and shed additional light on GIST biology. This will contribute to the continuing progress against this entity.

\section{Acknowledgment}

SMP is supported by the Sarcoma Alliance for Research through Collaboration (SARC) Career Development Award, the Sarcoma Foundation for America, 1K23CA175167-01, and the Gilman Sarcoma Foundation.

\section{Disclosure}

The authors report no conflicts of interest in this work.

\section{References}

1. Corless CL, Fletcher JA, Heinrich MC. Biology of gastrointestinal stromal tumors. J Clin Oncol. 2004;22(18):3813-3825.

2. Plesec TP. Gastrointestinal mesenchymal neoplasms other than gastrointestinal stromal tumors: focusing on their molecular aspects. Patholog Res Int. 2011;2011:952569.

3. Miettinen M, Lasota J. Gastrointestinal stromal tumors. Gastroenterol Clin North Am. 2013;42(2):399-415.
4. Rajendra R, Pollack SM, Jones RL. Management of gastrointestinal stromal tumors. Future Oncol. 2013;9(2):193-206.

5. Chou FF, Eng HL, Sheen-Chen SM. Smooth muscle tumors of the gastrointestinal tract: analysis of prognostic factors. Surgery. 1996;119(2): 171-177.

6. Hirota S, Isozaki K, Nishida T, Kitamura Y. Effects of loss-of-function and gain-of-function mutations of c-kit on the gastrointestinal tract. J Gastroenterol. 2000;35 Suppl 12:75-79.

7. Corless CL, Heinrich MC. Molecular pathobiology of gastrointestinal stromal sarcomas. Annu Rev Pathol. 2008;3:557-586.

8. Medeiros F, Corless CL, Duensing A, et al. KIT-negative gastrointestinal stromal tumors: proof of concept and therapeutic implications. Am J Surg Pathol. 2004;28(7):889-894.

9. Heinrich MC, Corless CL, Duensing A, et al. PDGFRA activating mutations in gastrointestinal stromal tumors. Science. 2003;299(5607): 708-710.

10. Liegl B, Hornick JL, Corless CL, Fletcher CD. Monoclonal antibody DOG1.1 shows higher sensitivity than KIT in the diagnosis of gastrointestinal stromal tumors, including unusual subtypes. Am J Surg Pathol. 2009;33(3):437-446.

11. Hohenberger P, Ronellenfitsch U, Oladeji O, et al. Pattern of recurrence in patients with ruptured primary gastrointestinal stromal tumour. Br J Surg. 2010;97(12):1854-1859.

12. Hirota S, Isozaki K, Moriyama Y, et al. Gain-of-function mutations of c-kit in human gastrointestinal stromal tumors. Science. 1998;279(5350): 577-580.

13. Singer S, Rubin BP, Lux ML, et al. Prognostic value of KIT mutation type, mitotic activity, and histologic subtype in gastrointestinal stromal tumors. J Clin Oncol. 2002;20(18):3898-3905.

14. Sommer G, Agosti V, Ehlers I, et al. Gastrointestinal stromal tumors in a mouse model by targeted mutation of the kit receptor tyrosine kinase. Proc Natl Acad Sci U S A. 2003;100(11):6706-6711.

15. Lasota J, Jasinski M, Sarlomo-Rikala M, Miettinen M. Mutations in exon 11 of c-Kit occur preferentially in malignant versus benign gastrointestinal stromal tumors and do not occur in leiomyomas or leiomyosarcomas. Am J Pathol. 1999;154(1):53-60.

16. Miettinen M, Furlong M, Sarlomo-Rikala M, Burke A, Sobin LH, Lasota J. Gastrointestinal stromal tumors, intramural leiomyomas, and leiomyosarcomas in the rectum and anus: a clinicopathologic, immunohistochemical, and molecular genetic study of 144 cases. Am J Surg Pathol. 2001;25(9):1121-1133.

17. Gutierrez JC, De Oliveira LO, PerezEA, Rocha-Lima C, Livingstone AS, Koniaris LG. Optimizing diagnosis, staging, and management of gastrointestinal stromal tumors. J Am Coll Surg. 2007;205(3):479-491 (quiz 524).

18. Joensuu H, Roberts PJ, Sarlomo-Rikala M, et al. Effect of the tyrosine kinase inhibitor STI571 in a patient with a metastatic gastrointestinal stromal tumor. N Engl J Med. 2001;344(14):1052-1056.

19. van Oosterom AT, Judson I, Verweij J, et al. Safety and efficacy of imatinib (STI571) in metastatic gastrointestinal stromal tumours: a phase I study. Lancet. 2001;358(9291):1421-1423.

20. Corless CL, Ballman KV, Antonescu CR, et al. Pathologic and molecular features correlate with long-term outcome after adjuvant therapy of resected primary GI stromal tumor: the ACOSOG Z9001 trial. J Clin Oncol. 2014;32(15):1563-1570.

21. Blay JY, Bonvalot S, Casali P, et al. Consensus meeting for the management of gastrointestinal stromal tumors. Report of the GIST consensus conference of 20-21 March 2004, under the auspices of ESMO. Ann Oncol. 2005;16(4):566-578.

22. Joensuu H, Eriksson M, Sundby Hall K, et al. One vs three years of adjuvant imatinib for operable gastrointestinal stromal tumor: a randomized trial. JAMA. 2012;307(12):1265-1272.

23. Dematteo RP, Ballman KV, Antonescu CR, et al. Adjuvant imatinib mesylate after resection of localised, primary gastrointestinal stromal tumour: a randomised, double-blind, placebo-controlled trial. Lancet. 2009;373(9669):1097-1104. 
24. Joensuu H, Hohenberger P, Corless CL. Gastrointestinal stromal tumour. Lancet. 2013;382(9896):973-983.

25. George S, Blay JY, Casali PG, et al. Clinical evaluation of continuous daily dosing of sunitinib malate in patients with advanced gastrointestinal stromal tumour after imatinib failure. Eur J Cancer. 2009;45(11):1959-1968.

26. Ricci R, Dei Tos AP, Rindi G. GISTogram: a graphic presentation of the growing GIST complexity. Virchows Arch. 2013;463(4):481-487.

27. Mazur MT, Clark HB. Gastric stromal tumors. Reappraisal of histogenesis. Am J Surg Pathol. 1983;7(6):507-519.

28. Nannini M, Astolfi A, Urbini M, et al. Integrated genomic study of quadruple-WT GIST (KIT/PDGFRA/SDH/RAS pathway wild-type GIST). BMC Cancer. 2014;14:685.

29. Lourenco N, Helias-Rodzewicz Z, Bachet JB, et al. Copy-neutral loss of heterozygosity and chromosome gains and losses are frequent in gastrointestinal stromal tumors. Mol Cancer. 2014;13:246.

30. Astolfi A, Nannini M, Pantaleo MA, et al. A molecular portrait of gastrointestinal stromal tumors: an integrative analysis of gene expression profiling and high-resolution genomic copy number. Lab Invest. 2010; 90(9):1285-1294.

31. Dewaele B, Wasag B, Cools J, et al. Activity of dasatinib, a dual SRC/ ABL kinase inhibitor, and IPI-504, a heat shock protein 90 inhibitor, against gastrointestinal stromal tumor-associated PDGFRAD842V mutation. Clin Cancer Res. 2008;14(18):5749-5758.

32. Huynh H, Lee JW, Chow PK, et al. Sorafenib induces growth suppression in mouse models of gastrointestinal stromal tumor. Mol Cancer Ther. 2009;8(1):152-159.

33. Wilhelm SM, Dumas J, Adnane L, et al. Regorafenib (BAY 73-4506): a new oral multikinase inhibitor of angiogenic, stromal and oncogenic receptor tyrosine kinases with potent preclinical antitumor activity. Int J Cancer. 2011;129(1):245-255.

34. Mross K, Frost A, Steinbild S, et al. A phase I dose-escalation study of regorafenib (BAY 73-4506), an inhibitor of oncogenic, angiogenic, and stromal kinases, in patients with advanced solid tumors. Clin Cancer Res. 2012;18(9):2658-2667.

35. George S, Wang Q, Heinrich MC, et al. Efficacy and safety of regorafenib in patients with metastatic and/or unresectable GI stromal tumor after failure of imatinib and sunitinib: a multicenter phase II trial. J Clin Oncol. 2012;30(19):2401-2407.

36. Yang JC, Haworth L, Sherry RM, et al. A randomized trial of bevacizumab, an anti-vascular endothelial growth factor antibody, for metastatic renal cancer. N Engl J Med. 2003;349(5):427-434.

37. Hurwitz H, Fehrenbacher L, Novotny W, et al. Bevacizumab plus irinotecan, fluorouracil, and leucovorin for metastatic colorectal cancer. N Engl J Med. 2004;350(23):2335-2342.

38. Napolitano S, Martini G, Rinaldi B, et al. Primary and acquired resistance of colorectal cancer to anti-EGFR monoclonal antibody can be overcome by combined treatment of regorafenib with cetuximab. Clin Cancer Res. 2015;21(13):2975-2983.

39. Van Looy T, Gebreyohannes YK, Wozniak A, et al. Characterization and assessment of the sensitivity and resistance of a newly established human gastrointestinal stromal tumour xenograft model to treatment with tyrosine kinase inhibitors. Clin Sarcoma Res. 2014;4:10.

40. Kim TS, Cavnar MJ, Cohen NA, et al. Increased KIT inhibition enhances therapeutic efficacy in gastrointestinal stromal tumor. Clin Cancer Res. 2014;20(9):2350-2362.

41. Carr BI, Cavallini A, Lippolis C, et al. Fluoro-Sorafenib (Regorafenib) effects on hepatoma cells: growth inhibition, quiescence, and recovery. J Cell Physiol. 2013;228(2):292-297.

42. D'Alessandro R, Refolo MG, Lippolis C, et al. Reversibility of regorafenib effects in hepatocellular carcinoma cells. Cancer Chemother Pharmacol. 2013;72(4):869-877.

43. Crona DJ, Keisler MD, Walko CM. Regorafenib: a novel multitargeted tyrosine kinase inhibitor for colorectal cancer and gastrointestinal stromal tumors. Ann Pharmacother. 2013;47(12):1685-1696.

44. Zierle J, Bissinger R, Bouguerra G, Abbes S, Lang F. Triggering of suicidal erythrocyte death by regorafenib. Cell Physiol Biochem. 2016; 38(1):160-172.
45. Weng Z, Luo Y, Yang X, et al. Regorafenib impairs mitochondrial functions, activates AMP-activated protein kinase, induces autophagy, and causes rat hepatocyte necrosis. Toxicology. 2015;327:10-21.

46. Heinrich MC, Maki RG, Corless CL, et al. Primary and secondary kinase genotypes correlate with the biological and clinical activity of sunitinib in imatinib-resistant gastrointestinal stromal tumor. J Clin Oncol. 2008; 26(33):5352-5359.

47. Li J, Qin S, Xu R, et al. Regorafenib plus best supportive care versus placebo plus best supportive care in Asian patients with previously treated metastatic colorectal cancer (CONCUR): a randomised, doubleblind, placebo-controlled, phase 3 trial. Lancet Oncol. 2015;16(6): 619-629.

48. Grothey A, Van Cutsem E, Sobrero A, et al. Regorafenib monotherapy for previously treated metastatic colorectal cancer (CORRECT): an international, multicentre, randomised, placebo-controlled, phase 3 trial. Lancet. 2013;381(9863):303-312.

49. van der Graaf WT, Blay JY, Chawla SP, et al. Pazopanib for metastatic soft-tissue sarcoma (PALETTE): a randomised, double-blind, placebocontrolled phase 3 trial. Lancet. 2012;379(9829):1879-1886.

50. Rajendra R, Jones RL, Pollack SM. Targeted treatment for advanced soft tissue sarcoma: profile of pazopanib. OncoTargets Ther. 2013;6: 217-222.

51. Brodowicz T, Liegl-Atzwager B, Tresch E, et al. Study protocol of REGOSARC trial: activity and safety of regorafenib in advanced soft tissue sarcoma: a multinational, randomized, placebo-controlled, phase II trial. BMC Cancer. 2015;15:127.

52. Strumberg D, Scheulen ME, Schultheis B, et al. Regorafenib (BAY 73-4506) in advanced colorectal cancer: a phase I study. Br JCancer. 2012; 106(11):1722-1727.

53. Demetri GD, Reichardt P, Kang YK, et al. Efficacy and safety of regorafenib for advanced gastrointestinal stromal tumours after failure of imatinib and sunitinib (GRID): an international, multicentre, randomised, placebo-controlled, phase 3 trial. Lancet. 2013;381(9863):295-302.

54. Wang Z, Xu J, Nie W, Huang G, Tang J, Guan X. Risk of hypertension with regorafenib in cancer patients: a systematic review and metaanalysis. Eur J Clin Pharmacol. 2014;70(2):225-231.

55. Jones RL, Bendell JC, Smith DC, et al. A phase I open-label trial evaluating the cardiovascular safety of regorafenib in patients with advanced cancer. Cancer Chemother Pharmacol. 2015;76(4):777-784.

56. Rutkowski P, Stepniak J. The safety of regorafenib for the treatment of gastrointestinal stromal tumors. Expert Opin Drug Saf. 2016;15(1): $105-116$.

57. Poole CD, Connolly MP, Chang J, Currie CJ. Health utility of patients with advanced gastrointestinal stromal tumors (GIST) after failure of imatinib and sunitinib: findings from GRID, a randomized, doubleblind, placebo-controlled phase III study of regorafenib versus placebo. Gastric Cancer. 2015;18(3):627-634.

58. Sawaki A, Nishida T, Doi T, et al. Phase 2 study of nilotinib as third-line therapy for patients with gastrointestinal stromal tumor. Cancer. 2011; 117(20):4633-4641.

59. Garner AP, Gozgit JM, Anjum R, et al. Ponatinib inhibits polyclonal drug-resistant KIT oncoproteins and shows therapeutic potential in heavily pretreated gastrointestinal stromal tumor (GIST) patients. Clin Cancer Res. 2014;20(22):5745-5755.

60. Cohen NA, Zeng S, Seifert AM, et al. Pharmacological inhibition of KIT activates MET signaling in gastrointestinal stromal tumors. Cancer Res. 2015;75(10):2061-2070.

61. Mahadevan D, Theiss N, Morales C, et al. Novel receptor tyrosine kinase targeted combination therapies for imatinib-resistant gastrointestinal stromal tumors (GIST). Oncotarget. 2015;6(4):1954-1966.

62. Li J, Dang Y, Gao J, Li Y, Zou J, Shen L. PI3K/AKT/mTOR pathway is activated after imatinib secondary resistance in gastrointestinal stromal tumors (GISTs). Med Oncol. 2015;32(4):111.

63. Floris G, Wozniak A, Sciot R, et al. A potent combination of the novel PI3K Inhibitor, GDC-0941, with imatinib in gastrointestinal stromal tumor xenografts: long-lasting responses after treatment withdrawal. Clin Cancer Res. 2013;19(3):620-630. 
64. Agaram NP, Wong GC, Guo T, et al. Novel V600E BRAF mutations in imatinib-naive and imatinib-resistant gastrointestinal stromal tumors. Genes Chromosomes Cancer. 2008;47(10):853-859.

65. Singeltary B, Ghose A, Sussman J, Choe K, Olowokure O. Durable response with a combination of imatinib and sorafenib in KIT exon 17 mutant gastrointestinal stromal tumor. J Gastrointest Oncol. 2014;5(1): E27-E29.

66. Edris B, Willingham SB, Weiskopf K, et al. Anti-KIT monoclonal antibody inhibits imatinib-resistant gastrointestinal stromal tumor growth. Proc Natl Acad Sci U S A. 2013;110(9):3501-3506.
67. Chen LL, Gouw L, Sabripour M, Hwu WJ, Benjamin RS. Combining targeted therapy with immunotherapy (interferon-alpha): rational, efficacy in gastrointestinal stromal tumor model and implications in other malignancies. Oncoimmunology. 2012;1(5):773-776.

68. Balachandran VP, Cavnar MJ, Zeng S, et al. Imatinib potentiates antitumor $\mathrm{T}$ cell responses in gastrointestinal stromal tumor through the inhibition of Ido. Nat Med. 2011;17(9):1094-1100.

\section{Publish your work in this journal}

OncoTargets and Therapy is an international, peer-reviewed, open access journal focusing on the pathological basis of all cancers, potential targets for therapy and treatment protocols employed to improve the management of cancer patients. The journal also focuses on the impact of management programs and new therapeutic agents and protocols on

\section{Dovepress}

patient perspectives such as quality of life, adherence and satisfaction. The manuscript management system is completely online and includes a very quick and fair peer-review system, which is all easy to use. Visit http://www.dovepress.com/testimonials.php to read real quotes from published authors.

Submit your manuscript here: http://www.dovepress.com/oncotargets-and-therapy-journal 